\title{
Locus coeruleus integrity and the effect of atomoxetine on response inhibition in Parkinson's disease
}

\author{
(D)Claire O'Callaghan, ${ }^{1,2, \dagger}$ (DFrank H. Hezemans, ${ }^{3,4, \dagger}$ Rong Ye, ${ }^{4}$ Catarina Rua, ${ }^{4,5}$ \\ P. Simon Jones, ${ }^{4}$ (1)Alexander G. Murley, ${ }^{4}$ Negin Holland, ${ }^{4}$ Ralf Regenthal, ${ }^{6}$ \\ (1)Kamen A. Tsvetanov, ${ }^{4}$ Noham Wolpe, ${ }^{2,7}$ Roger A. Barker, ${ }^{8,9}$ \\ Caroline H. Williams-Gray, ${ }^{8}$ Trevor W. Robbins, ${ }^{10,11}$ (DLuca Passamonti ${ }^{4}$ and \\ (D)James B. Rowe $\mathrm{R}^{3,4,12}$
}

${ }^{\dagger}$ These authors contributed equally to this work.

Cognitive decline is a common feature of Parkinson's disease, and many of these cognitive deficits fail to respond to dopaminergic therapy. Therefore, targeting other neuromodulatory systems represents an important therapeutic strategy. Among these, the locus coeruleus-noradrenaline system has been extensively implicated in response inhibition deficits. Restoring noradrenaline levels using the noradrenergic reuptake inhibitor atomoxetine can improve response inhibition in some patients with Parkinson's disease, but there is considerable heterogeneity in treatment response. Accurately predicting the patients who would benefit from therapies targeting this neurotransmitter system remains a critical goal, in order to design the necessary clinical trials with stratified patient selection to establish the therapeutic potential of atomoxetine.

Here, we test the hypothesis that integrity of the noradrenergic locus coeruleus explains the variation in improvement of response inhibition following atomoxetine. In a double-blind placebo-controlled randomized crossover design, 19 patients with Parkinson's disease completed an acute psychopharmacological challenge with $40 \mathrm{mg}$ of oral atomoxetine or placebo. A stop-signal task was used to measure response inhibition, with stop-signal reaction times obtained through hierarchical Bayesian estimation of an ex-Gaussian race model. Twenty-six control subjects completed the same task without undergoing the drug manipulation. In a separate session, patients and controls underwent ultra-high field $7 \mathrm{~T}$ imaging of the locus coeruleus using a neuromelanin-sensitive magnetization transfer sequence.

The principal result was that atomoxetine improved stop-signal reaction times in those patients with lower locus coeruleus integrity. This was in the context of a general impairment in response inhibition, as patients on placebo had longer stop-signal reaction times compared to controls. We also found that the caudal portion of the locus coeruleus showed the largest neuromelanin signal decrease in the patients compared to controls.

Our results highlight a link between the integrity of the noradrenergic locus coeruleus and response inhibition in patients with Parkinson's disease. Furthermore, they demonstrate the importance of baseline noradrenergic state in determining the response to atomoxetine. We suggest that locus coeruleus neuromelanin imaging offers a marker of noradrenergic capacity that could be used to stratify patients in trials of noradrenergic therapy and to ultimately inform personalized treatment approaches. 
1 Brain and Mind Centre and School of Medical Sciences, Faculty of Medicine and Health, University of Sydney, Sydney 2050, Australia

2 Department of Psychiatry, University of Cambridge, Cambridge CB2 OSZ, UK

3 MRC Cognition and Brain Sciences Unit, University of Cambridge, Cambridge CB2 7EF, UK

4 Department of Clinical Neurosciences, University of Cambridge, Cambridge CB2 OSZ, UK

5 Wolfson Brain Imaging Centre, University of Cambridge, Cambridge 04107, UK

6 Division of Clinical Pharmacology, Rudolf-Boehm-Institute for Pharmacology and Toxicology, University of Leipzig, Leipzig 69978, Germany

7 Department of Physical Therapy, Faculty of Medicine, Tel Aviv University, Tel Aviv, Israel

8 John van Geest Centre for Brain Repair, Department of Clinical Neurosciences, University of Cambridge, Cambridge CB2 OSZ, UK

9 Wellcome Trust-Medical Research Council Stem Cell Institute, University of Cambridge, Cambridge CB2 OAW, UK

10 Department of Psychology, University of Cambridge, Cambridge CB2 3EA, UK

11 Behavioural and Clinical Neuroscience Institute, University of Cambridge, Cambridge CB2 3EA, UK

12 Cambridge University Hospitals NHS Trust, Cambridge, CB2 OQQ UK

Correspondence to: Claire O'Callaghan

Brain and Mind Centre, 94 Mallett Street

Camperdown, NSW 2050, Australia

E-mail: claire.ocallaghan@sydney.edu.au

Correspondence may also be addressed to: Frank H. Hezemans

MRC Cognition and Brain Sciences Unit, 15 Chaucer Road

Cambridge CB2 7EF, UK

E-mail: frankhezemans@gmail.com

Keywords: Parkinson's disease; noradrenaline; atomoxetine; locus coeruleus; neuromelanin imaging Abbreviations: $\mathrm{BF}$ = Bayes factor; $\mathrm{CNR}=$ contrast-to-noise ratio; $\mathrm{MT}$ = magnetization transfer; $\mathrm{SSRT}$ = stop-signal reaction time

\section{Introduction}

Cognitive decline in Parkinson's disease remains an ongoing therapeutic challenge. The mainstay dopaminergic therapies often fail to improve cognitive deficits, and in some cases can exacerbate them. ${ }^{1-3}$ This has prompted a shift of focus towards other neuromodulatory systems that are affected by Parkinson's disease and related to cognitive decline, including noradrenaline. ${ }^{4}$ The noradrenergic locus coeruleus is one of the earliest sites of alpha-synuclein pathology, ${ }^{5-7}$ and noradrenergic treatments have been shown to modulate cognitive functions that are impaired in Parkinson's disease, including response inhibition. ${ }^{8-12}$

Response inhibition deficits are a well-documented feature of Parkinson's disease, ranging from subclinical impairments to extreme impulsive behaviours. ${ }^{13-17}$ Impulsivity is clear in the florid 'impulse control disorders' that are exacerbated by dopaminergic therapy. ${ }^{18}$ However, milder impulsivity is common in the absence of an impulse control disorder, including impairments in the ability to cancel an inappropriate action. Neurodegeneration of frontostriatal circuits, including the subthalamic nucleus and its inputs, contributes to this impairment in Parkinson's disease, ${ }^{15,19-21}$ while pharmacological modulation of these circuits offers a tractable route to restorative treatment. ${ }^{10-12,22}$

The locus coeruleus-noradrenaline system modulates the stimulus detection and behavioural reorienting required for rapid action cancellation. ${ }^{23,24}$ Phasic activation in the locus coeruleusthe brain's main source of noradrenaline-occurs in response to salient events, and its activity is tightly time-locked to task-relevant responses. ${ }^{25}$ Highly collateralized projections from the locus coeruleus enable release of noradrenaline in multiple brain regions, altering the gain, or responsivity, of target neurons. The action of noradrenaline at multiple targets can interrupt and reconfigure network architecture, promoting a change in goaldirected behaviour. ${ }^{26,27}$ This locus coeruleus-noradrenaline function directly supports rapid action cancellation. In healthy adults and in preclinical models, pharmacologically increasing noradrenaline levels with the reuptake inhibitor atomoxetine improves action cancellation, as measured on stop-signal tasks. ${ }^{28-31}$

Atomoxetine selectively inhibits presynaptic noradrenaline transporters, resulting in a 3-fold increase in extracellular levels of noradrenaline in the prefrontal cortex. ${ }^{32}$ It is currently licensed for treating behavioural and cognitive symptoms associated with attention deficit hyperactivity disorder. However, experimental psychopharmacological studies indicate it may be of value in some patients with Parkinson's disease, by increasing activity and connectivity in the fronto-striatal 'stopping network'. ${ }^{12,30,31}$ The stopping network includes the inferior frontal gyrus and presupplementary motor area, and their excitatory connection with the subthalamic nucleus which, via the globus pallidus, increases inhibition over thalamocortical output. ${ }^{33,34}$ Within this network, noradrenaline increases cortical excitability, ${ }^{35}$ functional connectivity ${ }^{36}$ and network integration. ${ }^{37}$ The prefrontal regions also provide descending input to modulate the locus coeruleus, ${ }^{38,39}$ by which prefrontal noradrenaline can influence locus coeruleus activity. Atomoxetine alters locus coeruleus firing patterns to increase the phasic-to-tonic ratio, making the locus coeruleus more responsive to task-relevant stimuli. ${ }^{40}$

The potential for atomoxetine to modulate locus coeruleusnoradrenaline function and improve response inhibition holds therapeutic promise in Parkinson's disease. Previous work using 
stop-signal tasks in Parkinson's disease demonstrated that atomoxetine can improve response inhibition and enhance its attendant stopping network activation. ${ }^{10-12,41}$ However, there was considerable heterogeneity in treatment response. To accurately predict the patients who would benefit from noradrenergic therapy remains a critical goal for atomoxetine treatment to be considered therapeutically and to design the necessary clinical trials with stratified patient selection. ${ }^{41,42}$

Here, we test the hypothesis that structural integrity of the noradrenergic locus coeruleus explains the variation in improvements in response inhibition following atomoxetine. This is now possible through recent developments in ultra-high field $7 \mathrm{~T}$ imaging of the locus coeruleus. ${ }^{43-45}$ We tested this hypothesis by combining quantification of the locus coeruleus by 7 T MRI with an acute psychopharmacological challenge, and measuring response inhibition using the stop-signal reaction time (SSRT).

\section{Materials and methods}

\section{Participants}

Nineteen patients with idiopathic Parkinson's disease were recruited via the University of Cambridge Parkinson's disease research clinic and the Parkinson's UK volunteer network. They met the United Kingdom Parkinson's Disease Society Brain Bank criteria and were not demented based on Movement Disorders Society (MDS) criteria for Parkinson's disease dementia ${ }^{46}$ nor on the MiniMental State Examination (MMSE, score > 26). ${ }^{47}$ They were aged between 50 and 80 years, with Hoehn and Yahr stages 1.5-3, and had no contraindications to $7 \mathrm{~T}$ MRI or atomoxetine. None had current impulse control disorders, based on clinical impression and the Questionnaire for Impulsive-Compulsive Disorders in Parkinson's Disease (QUIP-Current Short) screening tool. ${ }^{48}$ Levodopa equivalent daily dose (LEDD) scores were calculated. ${ }^{49}$ Twenty-six age-, sex- and education-matched healthy control subjects were recruited from local volunteer panels. Control participants were screened for a history of neurological or psychiatric disorders, and no controls were using psychoactive medications. The study was approved by the local Ethics Committees and all participants provided written informed consent according to the Declaration of Helsinki. Demographic details and clinical characteristics are provided in Table 1 and the Supplementary material.

\section{Study procedure}

Participants with Parkinson's disease were tested across three sessions. First, they underwent MRI scanning and clinical assessment, including the Movement Disorders Society Unified Parkinson's Disease Rating Scale (MDS-UPDRS), ${ }^{50}$ MMSE, Montreal Cognitive Assessment (MoCA $)^{51}$ and the revised Addenbrooke's Cognitive Examination (ACE-R). ${ }^{52}$

On the second and third sessions, patients completed a doubleblind randomized placebo-controlled crossover study, with $40 \mathrm{mg}$ of oral atomoxetine or placebo. Atomoxetine $40 \mathrm{mg}$ was chosen as this is at the lower end of the clinical dose range and very well tolerated, ${ }^{53,54}$ and previous studies have confirmed this dose is sufficient to modulate behaviour and functional brain activation in patients with Parkinson's disease. ${ }^{10-12}$ Drug/placebo order was randomly permuted in groups of six successive recruits; that is, we randomized three participants to placebo and three to atomoxetine within each successive block of 1-6, 7-12, 13-18 and 19-24. This strategy guards against unequal randomization that can arise if one were to randomize across the entire group. The visits were $\geqslant 6$ days apart [mean 7.4 days; standard deviation (SD) 1.7 days; range 6-14 days]. Blood samples were taken $2 \mathrm{~h}$ after administration of drug/placebo, to coincide with predicted peak

Table 1 Demographics and clinical assessments of participants in their normal ON medication state

\begin{tabular}{|c|c|c|c|c|}
\hline & $\begin{array}{l}\text { Parkinson's } \\
\text { disease }\end{array}$ & Controls & $\mathrm{BF}$ & $P$ \\
\hline Age, years & $67.11(7.05)$ & $65.35(5.32)$ & 0.43 & 0.368 \\
\hline Education, years & $14.05(2.27)$ & $14.65(3.10)$ & 0.37 & 0.457 \\
\hline Male/female & $15 / 4$ & $15 / 11$ & 0.98 & 0.240 \\
\hline MMSE & $29.47(0.70)$ & $29.77(0.51)$ & 0.87 & 0.128 \\
\hline MoCA & $28.11(1.76)$ & $28.58(1.39)$ & 0.45 & 0.340 \\
\hline \multicolumn{5}{|l|}{ ACE-R } \\
\hline Total score & $94.89(3.71)$ & $97.58(3.16)$ & 4.16 & 0.015 \\
\hline $\begin{array}{l}\text { Attention and } \\
\text { orientation }\end{array}$ & $17.84(0.37)$ & $17.96(0.20)$ & 0.64 & 0.216 \\
\hline Memory & $23.68(1.97)$ & $25.04(1.18)$ & 7.00 & 0.013 \\
\hline Fluency & $12.00(2.08)$ & $12.81(1.60)$ & 0.71 & 0.167 \\
\hline Language & $25.84(0.50)$ & $25.88(0.43)$ & 0.31 & 0.768 \\
\hline Visuospatial & $15.63(0.50)$ & $15.81(0.63)$ & 0.45 & 0.302 \\
\hline \multicolumn{5}{|l|}{ MDS-UPDRS } \\
\hline $\begin{array}{l}\text { I: Non-motor } \\
\text { experiences }\end{array}$ & $9.00(4.18)$ & & & \\
\hline II: Motor experiences & $12.63(4.26)$ & & & \\
\hline III: Motor examination & $28.42(11.60)$ & & & \\
\hline $\begin{array}{l}\text { IV: Motor } \\
\text { complications }\end{array}$ & $0.47(0.96)$ & & & \\
\hline Total score & $50.58(17.20)$ & & & \\
\hline Hoehn and Yahr stage & $2.26(0.45)$ & & & \\
\hline Disease duration, years & $4.15(1.72)$ & & & \\
\hline $\begin{array}{r}\text { Levodopa equivalent } \\
\text { daily dose, } \mathrm{mg} / \text { day }\end{array}$ & $644.55(492.81)$ & & & \\
\hline
\end{tabular}

Data are presented as mean (SD). Comparisons of patient and control groups were performed with independent samples t-tests or contingency tables as appropriate. ACE- $\mathrm{R}$ = revised Addenbrooke's Cognitive Examination; BF = Bayes factor where $>3$ indicates substantial evidence of a group difference; MDS-UPDRS $=$ Movement Disorders Society Unified Parkinson's Disease Rating Scale; MoCA = Montreal Cognitive Assessment; $P$ = $P$-value of classical two-sample t-test.

plasma concentration of atomoxetine after a single oral dose. ${ }^{55}$ Mean plasma concentration ${ }^{56}$ was $261.32 \mathrm{ng} / \mathrm{ml}$ after atomoxetine (SD $117.33 \mathrm{ng} / \mathrm{ml}$, range $90.92-595.11 \mathrm{ng} / \mathrm{ml}$ ) and $0 \mathrm{ng} / \mathrm{ml}$ after placebo. After the blood sample, patients commenced an experimental task battery that included a stop-signal response inhibition task. Supine/lying and upright blood pressure and pulse rate measures were monitored three times across the session (on arrival, $2 \mathrm{~h}$ post tablet administration, and on completion of testing). To monitor any changes in subjective feelings following the drug/placebo, prior to tablet administration and $2 \mathrm{~h}$ post, we administered a set of 16 visual analogue scales (VAS) rating current mood and arousal levels. All sessions and MRI scanning were conducted with patients on their regular anti-parkinsonian medications and at a similar time of day.

Control participants were tested in one session to provide normative data on the task, in which they underwent MRI scanning and completed the same experimental task battery as the patients. The control group did not undergo the drug/placebo manipulation. Both the patient and control groups completed a set of self- and informant-rated questionnaires to assess mood and behaviour, which are reported in detail in the Supplementary material.

\section{Stop-signal task}

We used a stop-signal task to measure response inhibition. This paradigm involves a two-choice reaction time 'go' task that 
A go no-go stop
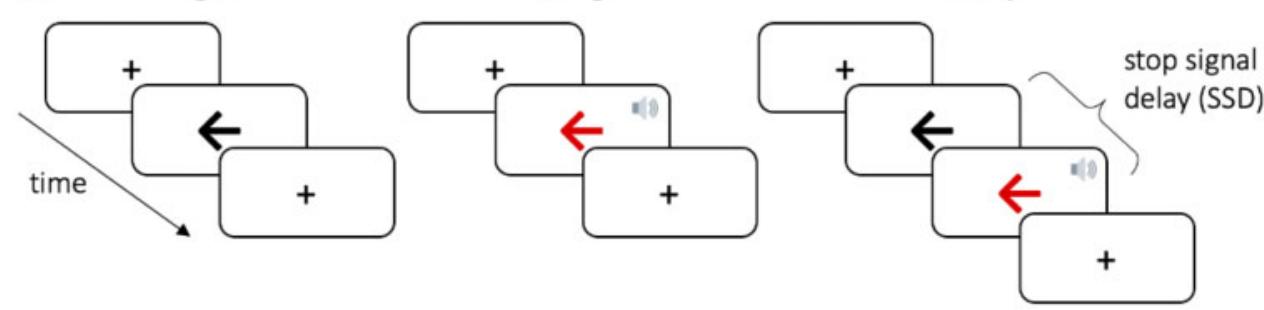

B

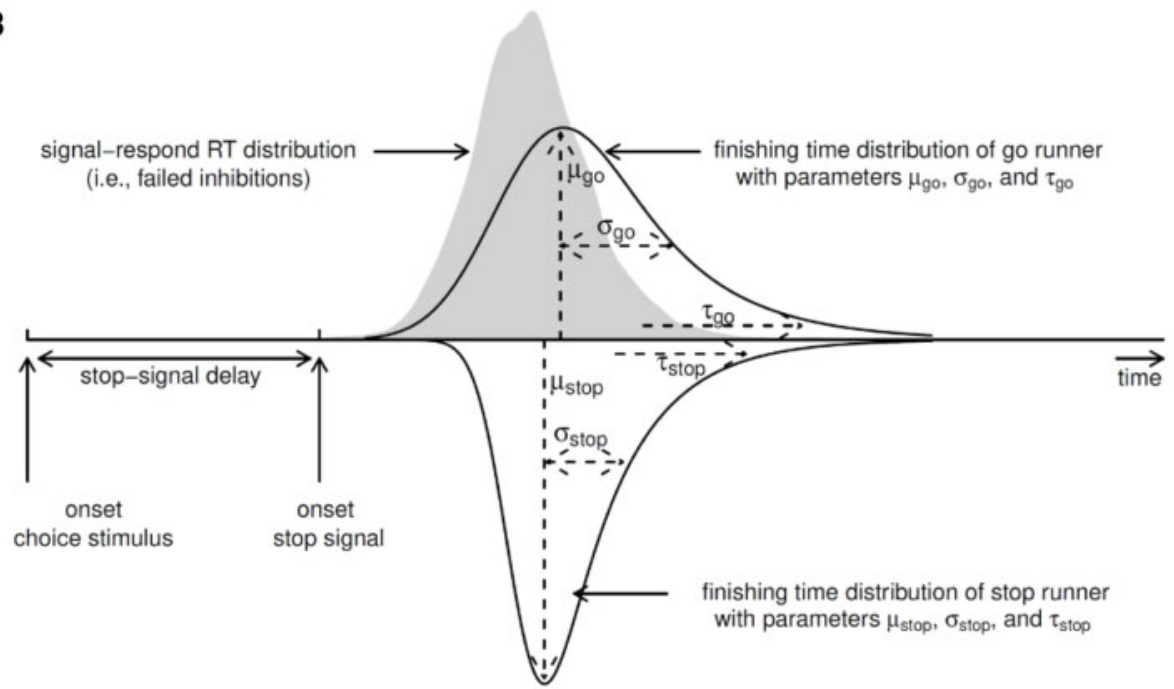

Figure 1 Design of the stop-signal task and ex-Gaussian race model of response inhibition. (A) In the stop-signal go/no-go task, participants respond as quickly and accurately as possible to the direction of a black arrow (go trials). Occasionally, this task is interrupted by a stop-signal (red arrow and beep tone), which requires any imminent response to be inhibited. For no-go trials, the stop-signal is presented immediately after the fixation cross. For stop trials, the stop-signal is presented after an initial go stimulus, with a short and variable delay. (B) The ex-Gaussian race model characterizes task performance as a race between three competing processes or 'runners': One stop process, and two go processes that match or mismatch the go stimulus. The finishing times of each process are assumed to follow an ex-Gaussian distribution. Successful inhibition in stop trials occurs when the stop process finishes before both go processes. A correct go response occurs when the matching go process finishes before the mismatching go process. For simplicity, the finishing time distribution of the mismatching go process is not illustrated. RT = reaction time. The speaker symbol in Fig. $1 \mathrm{~A}$ was copied from the Twitter emoji library, available at https://twemoji.twitter.com/ under a CC-BY 4.0 license (https://creativecommons.org/licenses/ by/4.0/). Figure 1B is from Heathcote et al., ${ }^{57}$ and is available at https://flic.kr/p/24g3sip under a CC-BY 2.0 license (https://creativecommons.org/ licenses/by/2.0/).

is occasionally interrupted by a 'stop signal', which requires the initiated response to be cancelled (Fig. 1A). On go trials, a left- or right-pointing black arrow was presented on the screen, and participants indicated its orientation by pressing a left or right response button. On stop trials, the arrow changed colour from black to red at the same time as a tone (i.e. the stop-signal), after a short and variable delay (i.e. the stop-signal delay, SSD). Participants were instructed to inhibit any imminent response if the arrow became red. The length of the SSD was varied across stop trials using a staircase method to target a stop accuracy of $50 \%$. The SSD ranged from $50 \mathrm{~ms}$ to $1500 \mathrm{~ms}$ and increased or decreased by $50 \mathrm{~ms}$ after a successful or failed stop trial, respectively. On no-go trials, the SSD was set to zero. The task consisted of four blocks of 140 trials each, including 110 go trials, 10 no-go trials (very low commission error rate), and 20 stop trials per block ( $50 \%$ commission error rate). The first 20 trials of each block were go trials, to compute a starting value for the SSD (mean reaction time $-200 \mathrm{~ms}$ ). The remaining trials within each block were pseudorandomly interleaved, with the constraints that there could be no more than seven consecutive go trials, and no more than two consecutive nogo or stop trials. At the start of each trial, a fixation cross was presented for $500 \mathrm{~ms}$.

Participants were given standardized instructions at the start of the experiment. They were asked to respond as 'quickly and accurately as possible', and were discouraged from strategically slowing down in anticipation of a stop signal. ${ }^{58}$ After these instructions, they were given a practice block of 25 trials ( 20 go trials, two no-go trials, and three stop trials). The experimenters verified that the participant understood the task, and if necessary, the practice block was repeated. The practice data were not analysed further. More extensive details about the task, conforming with the 'check list for reporting stop-signal studies' advised by Verbruggen et al., ${ }^{58}$ can be found in the Supplementary material.

\section{Ex-Gaussian race model of response inhibition}

We used a Bayesian parametric model of the stop-signal task to infer the latency of the unobservable stop response-the SSRT. ${ }^{58}$ This model assumes a race between three independent processes: one corresponding to the stop process, and two corresponding to go processes that match or mismatch the go stimulus (Fig. 1B). For a given stop trial, successful inhibition occurs when the stop process finishes before both go processes. For a given go trial, a correct response occurs when the matching go process finishes before the mismatching go process. The finish time distribution of the stop process is inferred by estimating the reaction time distribution of unsuccessful stop trials (i.e. signal respond reaction times). Specifically, the signal respond reaction time distribution is 
assumed to be a right-censored go reaction time distribution, where the censoring point for a given stop trial is drawn from the finish time distribution of the stop process (see Matzke et al. ${ }^{59}$ for details).

The model assumes that the finish times of the stop and go processes follow an ex-Gaussian distribution, which is a positively skewed unimodal distribution that is commonly used to describe reaction time data. ${ }^{60,61}$ Thus, for each process, we estimated the three parameters of the ex-Gaussian distribution: The mean $(\mu)$ and standard deviation $(\sigma)$ of the Gaussian component, and the mean (i.e. inverse rate) $(\tau)$ of the exponential component.

We additionally estimated two parameters that represent the probability that the stop and go processes failed to start, referred to as 'trigger failure' and 'go failure', respectively. ${ }^{62}$ These attentional failures are common in both healthy participants ${ }^{63,64}$ and in clinical cohorts, ${ }^{65,66}$ and if not modelled can severely bias estimation of the stop process. ${ }^{62,64,67}$ Prior to fitting the model, we removed implausibly short $(<0.25 \mathrm{~s})$ or long $(>4.5 \mathrm{~s})$ reaction times, as well as go reaction times more extreme than $\pm 2.5 \mathrm{SD}$ from the participant's mean. ${ }^{59}$

We used Markov chain Monte Carlo (MCMC) sampling to estimate the posterior distributions of the parameters. The parameters were estimated hierarchically, such that parameters for a given participant were sampled from corresponding group-level distributions. We fitted this hierarchical model separately for the control group, the Parkinson's disease group on placebo, and the Parkinson's disease group on atomoxetine. We placed the same set of prior distributions on the group-level parameters for each of these three groups. The prior distributions were identical to those suggested by the model developers, ${ }^{57}$ except for slightly higher prior mean values for $\mu_{\text {go-match }}(1.5 \mathrm{~s}), \mu_{\text {go-mismatch }}(1.5 \mathrm{~s})$ and $\mu_{\text {stop }}$ (1s), to account for slower reaction times in older age (see the Supplementary material for a full list of priors). The model MCMC sampling initially ran with 33 chains (i.e. three times the number of parameters), with thinning of every 10th sample and a 5\% probability of migration. Model convergence was assessed with the potential scale reduction statistic $\hat{R}(<1.1$ for all parameters), and with visual inspection of the time-series plots of the chains. After this, an additional 500 iterations for each chain were run to create a final posterior distribution for each parameter. To assess the model's goodness of fit, the observed data were compared to simulated data generated from the model's posterior predictive distribution (Supplementary Figs 8-10).

The primary outcome of interest, SSRT, was computed as the mean of the ex-Gaussian finish time distribution of the stop process, which is given by $\mu_{\text {stop }}+\tau_{\text {stop. }}$. We repeated this for each MCMC sample to approximate a posterior distribution of SSRT. This approach was also used to approximate a posterior distribution of go reaction time $\left(\mu_{\text {go-match }}+\tau_{\text {go-match }}\right)$.

\section{Statistical analysis}

The go error rate and stop accuracy rate served as basic descriptive statistics for stop-signal task performance. We defined the go error rate as the proportion of go trials with an incorrect response, including commission errors (responses that mismatch the arrow direction) and omission errors (missing responses). The stop accuracy rate was defined as the proportion of stop trials with successfully inhibited responses (missing responses). For both outcomes, we examined differences between groups (Parkinson's disease placebo versus controls) and drug conditions (Parkinson's disease placebo versus atomoxetine) using independent and paired samples t-tests, respectively.

We then examined the group- and participant-level parameter estimates from the ex-Gaussian race model described above. For group-level inference, we examined the posterior distributions of the group-level means of SSRT and go reaction time. For a given posterior distribution, we took the median as the posterior estimate, and the $95 \%$ quantile interval (QI) as the range of most credible values. We also obtained posterior distributions for contrasts of interest (Parkinson's disease placebo versus controls; Parkinson's disease placebo versus atomoxetine) by subtracting the sets of MCMC samples of the two groups under consideration. That is, for a given parameter, we computed the difference between the two groups for each MCMC sample, thereby yielding an approximate posterior distribution of the difference. ${ }^{68}$

To test for individual differences in the effect of atomoxetine on SSRT and go reaction time, we extracted the medians of the participant-level posterior distributions of SSRT and go reaction time from the placebo and atomoxetine model fits. We hypothesized that the effect of atomoxetine would depend on the integrity of the locus coeruleus, indexed by the contrast-to-noise ratio (CNR; described below). Therefore, the posterior parameter estimates were entered as the dependent variable with drug condition (placebo versus atomoxetine), CNR, and their interaction as fixed effects, allowing the intercept to vary across participants (random effect). We additionally included a fixed effect of session (first versus second) as a covariate of no interest. Taking the analysis of SSRT as an example, the model was specified in R formula syntax as follows: SSRT $\sim$ drug $\times$ CNR + session $+(1 \mid$ subject $)$.

For linear models, we report both frequentist and Bayes factor (BF) analyses for hypothesis testing, with a significance threshold of $P=0.05$ (two-sided) for frequentist analyses. We present the BF for the alternative hypothesis over the null hypothesis (i.e. $\mathrm{BF}_{10}$ ), such that $\mathrm{BF}>1$ indicates relative evidence for the alternative hypothesis, and $\mathrm{BF}>3$ indicates 'positive evidence' for the alternative hypothesis. ${ }^{69}$ All BF analyses used the default 'JZS' prior on the effect size under the alternative hypothesis. ${ }^{70,71}$ To test for specific fixed effects in linear mixed models, we obtained P-values using the Kenward-Roger method, and BFs through Bayesian model averaging by estimating the change from prior to posterior inclusion odds (inclusion BF). In other words, this model-averaged $\mathrm{BF}$ indicates how much more likely the data are under model variants that include a given fixed effect, compared to model variants that exclude the fixed effect. ${ }^{72}$

Because of technical issues, stop-signal task data were missing for one patient's placebo session and for another patient's atomoxetine session. We nevertheless included these two participants in the linear mixed model analyses of the within-subject effect of atomoxetine, as participants were treated as a random effect. However, excluding these two participants did not meaningfully change any of the following results.

\section{Software and equipment}

The stop-signal task was implemented in MATLAB R2018b using the Psychophysics Toolbox extensions (Version 3). ${ }^{73}$ Participants responded using a two-button response box. The ex-Gaussian model fitting was performed with the Dynamic Models of Choice toolbox, ${ }^{57}$ implemented in $\mathrm{R}$ (version 3.6.1, R Core Team, 2019). Further statistical analyses in $\mathrm{R}$ used the 'tidyverse" ${ }^{14}$ and 'tidybayes ${ }^{, 75}$ packages for data organization and visualization, the 'afex' package ${ }^{76}$ for ANOVA and linear mixed model fitting with the 'emmeans' package ${ }^{77}$ used for post hoc comparisons, and the 'BayesFactor'78 and 'bayestestR' ${ }^{79}$ packages for BF analysis.

\section{MRI acquisition}

All patients and controls underwent MRI. Two controls were excluded from further imaging analysis because of incidental 
structural abnormalities. MRIs were acquired with a $7 \mathrm{~T}$ Magnetom Terra scanner (Siemens), using a 32-channel receive and circularly polarized single-channel transmit head coil (Nova Medical). We used a 3D high-resolution magnetization transfer-weighted turbo flash (MT-TFL) sequence for imaging the locus coeruleus (based on Priovoulos et al. ${ }^{43}$ ). One hundred and twelve axial slices were used to cover both the midbrain and the pontine regions. The sequence applied a train of 20 Gaussian-shape RF-pulses at 6.72 ppm off resonance, $420^{\circ}$ flip angle, followed by a turbo-flash readout (echo time $=4.08 \mathrm{~ms}$, repetition time $=1251 \mathrm{~ms}$, flip angle $=8^{\circ}$, voxel size $=0.4 \times 0.4 \times 0.5 \mathrm{~mm}^{3}, 6 / 8$ phase and slice partial Fourier, bandwidth $=140 \mathrm{~Hz} / \mathrm{px}$, no acceleration, $14.3 \%$ oversampling, acquisition time $\sim 7 \mathrm{~min}$ ). For each subject, the transmit voltage was adjusted based on the average flip angle in the central area of the pons obtained from a B1 pre-calibration scan. The MT-TFL sequence was repeated twice and averaged offline to improve signalto-noise ratio. An additional scan (MT-off) was acquired with the same parameters as above but without the off-resonance pulses. A high resolution isotropic $\mathrm{T}_{1}$-weighted MP2RAGE image was also acquired sagittally for anatomical coregistration using the UK7T Network harmonized protocol ${ }^{80}$ : echo time $=2.58 \mathrm{~ms}$, repetition time $=3500 \mathrm{~ms}$, bandwidth $=300 \mathrm{~Hz} / \mathrm{px}$, voxel size $=0.7 \times 0.7 \times$ $0.7 \mathrm{~mm}^{3}$, field of view $=224 \times 224 \times 157 \mathrm{~mm}^{3}$, acceleration factor $(\mathrm{A} \gg \mathrm{P})=3$, flip angles $=5 / 2^{\circ}$ and inversion times $=725 / 2150 \mathrm{~ms}$ for the first/second images.

\section{Image processing and coregistration pipeline}

Image processing and coregistration was based on the pipeline described in Ye et al. ${ }^{45}$ The Advanced Normalization Tools (ANTs v2.2.0) software and in-house MATLAB scripts were used for image preprocessing and the standardization of MT images. MT images were first $\mathrm{N} 4$ bias field corrected for spatial inhomogeneity (number of iterations at each resolution level: $50 \times 50 \times 30 \times 20$, convergence threshold: $1 \times 10^{-6}$, isotropic sizing for $b$-spline fitting: 200 $)^{81}$ then averaged using the customized antsMultivariateTemplateConstruction2 function for improvements in signal-to-noise ratio. The $\mathrm{T}_{1}$-weighted MP2RAGE data were generated offline from the complex images. ${ }^{80} \mathrm{~T}_{1}$-weighted skull-stripped images were obtained after tissue type segmentation and reconstruction using SPM12 (v7219) (http://www.fil.ion. ucl.ac.uk/spm/software/spm12/ accessed 15 July 2021).

The preprocessed MT-weighted and $\mathrm{T}_{1}$-weighted images were then entered into a $\mathrm{T}_{1}$-driven, cross modality coregistration pipeline to warp the individual MT and MT-off images to the isotropic
$0.5 \mathrm{~mm}$ ICBM152 (International Consortium for Brain Mapping) $\mathrm{T}_{1^{-}}$ weighted asymmetric template. ${ }^{82}$ The individual $\mathrm{T}_{1}$-weighted images were first coregistered to the MT image with rigid only transformation. The MT-off image was used as the intermediate step for bridging the two modalities because the MT-off image shares similar tissue-specific contrasts with both $\mathrm{T}_{1}$-weighted MP2RAGE and MT-on images.

In parallel, an unbiased study-wise $\mathrm{T}_{1}$-weighted structural template was created using individual skull-stripped $\mathrm{T}_{1}$-weighted images from all controls and patients. Native $\mathrm{T}_{1}$-weighted images were rigid and affine transformed, followed by a hierarchical non-linear diffeomorphic step at five levels of resolution, repeated by six runs to improve convergence. Max iterations for each resolution from the coarsest level to the full resolution were $100 \times$ $100 \times 70 \times 50 \times 20$ (shrink factors: $10 \times 6 \times 4 \times 2 \times 1$, smoothing factors: $5 \times 3 \times 2 \times 1 \times 0$ voxels, gradient step size: $0.1 \mathrm{~mm}$ ). Greedy Symmetric Normalization (SyN) was adopted for the transformation model of the deformation step. ${ }^{83}$ The resulting $\mathrm{T}_{1}$ weighted group template was then registered to the standard ICBM152 $\mathrm{T}_{1}$-weighted brain following the similar rigid-affine-SyN steps at four resolution levels (max iterations: $100 \times 70 \times 50 \times 50$, convergence threshold: $1 \times 10^{-6}$, shrink factors: $8 \times 4 \times 2 \times 1$, smoothing factors: $3 \times 2 \times 1 \times 0$ voxels). For all the above registration steps, cross-correlation was used for similarity metrics estimation as it performs better for linear and non-linear components during intra-modality registration. Four steps of deformations were estimated as follows (in order): MT-off to MT, $\mathrm{T}_{1}$-weighted to MT-off, $\mathrm{T}_{1}$-weighted to $\mathrm{T}_{1}$-weighted group template and $\mathrm{T}_{1}$ weighted group template to ICBM152 $\mathrm{T}_{1}$-weighted template. These parameters were then used as the road map for MT image standardization to the ICBM brain in one step. A trilinear interpolation method was selected to preserve the absolute location and relative contrast of the signal.

\section{Independent probabilistic locus coeruleus atlas creation}

To facilitate accurate extraction of the locus coeruleus signal we created a study-specific unbiased locus coeruleus atlas (Fig. 2A). To this end, we used an independent sample of 29 age- and education-matched healthy control subjects [13 female; age mean (SD) = 67 (8.2), age range $=52-84]$ collected under the same neuroimaging protocol. We used a validated pipeline for locus coeruleus atlas construction described in Ye et al. ${ }^{45}$ Briefly, for each axial slice on
A

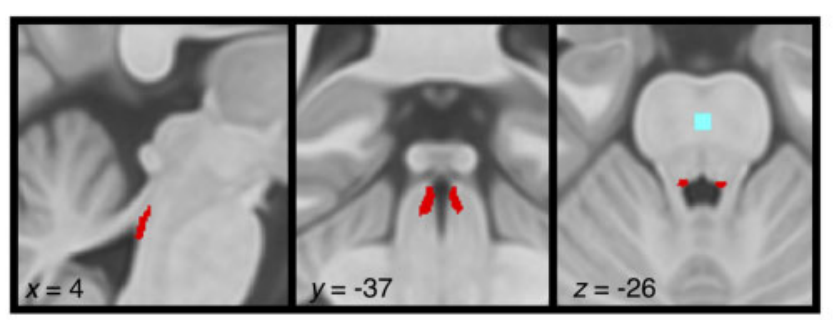

B

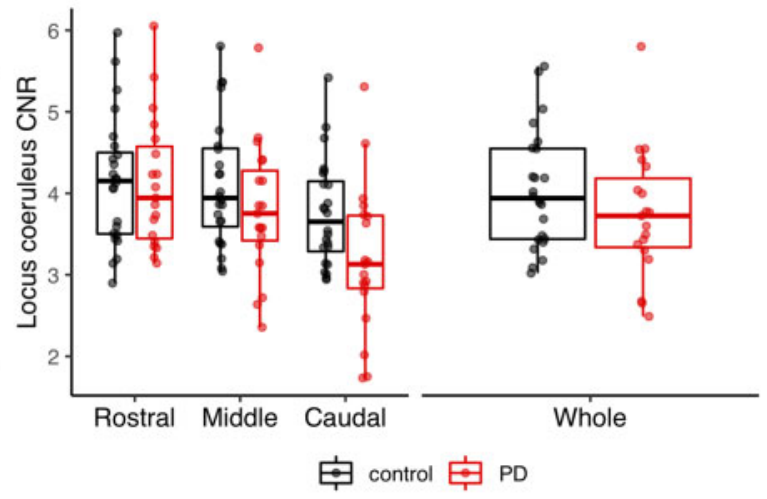

Figure 2 Locus coeruleus imaging. (A) Study specific locus coeruleus atlas, also showing the reference region (light blue) in the central pons. (B) CNR for the locus coeruleus subdivisions and whole structure in Parkinson's disease patients (PD) versus controls (note, left and right locus coeruleus are combined). 
the rostrocaudal extent, the locations of the left and right locus coeruleus were determined using a semi-automated segmentation method. A threshold was defined as 5 SD above the mean intensity in the central pontine reference region. After applying the threshold, locus coeruleus voxels on axial planes were automatically segmented into binarized images and then averaged to construct a probabilistic atlas. The independent locus coeruleus atlas generated for this study had very high similarity in the spatial distribution of probabilities and contours relative to the validated $7 \mathrm{~T}$ locus coeruleus atlas in Ye et al. ${ }^{45}$ (Supplementary Fig. 1).

\section{Locus coeruleus signal extraction}

As a measure of locus coeruleus integrity, we quantified contrast by establishing the CNR with respect to a reference region in the central pons (Fig. 2A). A CNR map was computed voxel-by-voxel on the average MT image for each subject using the signal difference between a given voxel $(\mathrm{V})$ and the mean intensity in the reference region $\left(\mathrm{Mean}_{\mathrm{REF}}\right)$ divided by the standard deviation $\left(\mathrm{SD}_{\mathrm{REF}}\right)$ of the reference signals:

$$
\left(\mathrm{CNR}=\frac{\mathrm{V}-\mathrm{Mean}_{\mathrm{REF}}}{\mathrm{SD}_{\mathrm{REF}}}\right)
$$

CNR values were extracted bilaterally on the CNR map by applying the independent locus coeruleus probabilistic atlas $(5 \%$ probability version). We computed mean CNR values for the rostral, middle and caudal portions of the left and right locus coeruleus. As an index of locus coeruleus integrity to incorporate with the stop-signal task analysis, we combined the left and right locus coeruleus and averaged across the whole structure.

\section{Data availability}

Code and data to reproduce manuscript figures, statistical analyses and stop-signal task modelling are freely available through the Open Science Framework (https://osf.io/tyka3/).

\section{Results}

\section{Behavioural results}

As shown in Table 1, the patient and control groups were matched in terms of age, years of education, sex ratio, MMSE and MoCA (all $P$-values > 0.050; all BFs $<1$ ). Patients had a significantly lower ACE-R total score $(P=0.015, B F=4.16)$, and lower memory subscale $(P=0.013, B F=7.00)$. There were no group differences on any of the self-reported questionnaires assessing impulsivity, anxiety, depression and behavioural measures, with the exception of the Motivation and Energy Inventory where the patient group had significantly lower scores for the physical subscale compared to controls $(P=0.010, B F=6.18$; Supplementary Table 1$)$. We did not see a bias in left versus right motor symptom laterality in the patient group; this analysis is described in detail in the Supplementary material.

Within the drug and placebo sessions, there was some evidence of increased pulse rates and raised blood pressure on atomoxetine, although this was not considered clinically relevant and was not consistently observed across all supine and upright measures. Importantly, there was no change in subjective ratings of mood and arousal levels within the sessions. These analyses are described in detail in the Supplementary material.

\section{Locus coeruleus integrity}

Figure 2B shows comparisons of locus coeruleus CNR between the patients and controls. When comparing across the whole structure, the groups were not significantly different $[t(36.98)=1.27$, $P=0.21, \mathrm{BF}=0.58]$. Comparing across the rostral, middle and caudal subdivisions, there was a main effect of subdivision $\left[F(1.27,52.22)=54.57, P<0.001 ; B F=5.28 \times 10^{10}\right]$. This was driven by CNR in the caudal portion being significantly lower than both the middle $[t(82)=7.37, P<0.0001]$ and rostral $[t(82)=10.10, P$ $<0.0001]$ portions. There was a significant group by subdivision interaction $[F(1.27,52.22)=7.89, P=004 ; B F=32.98]$. This reflected significantly lower CNR values in the caudal portion for patients relative to controls $[t(50.1)=2.23, P=0.026]$, whereas the groups did not differ for the middle $[t(50.1)=1.32, P=0.193]$ or rostral $[t(50.1)=0.140, P=0.889]$ portions of the locus coeruleus. We did not see significant differences in left versus right locus coeruleus degeneration, as the group comparisons did not meaningfully change when incorporating locus coeruleus side; this analysis is reported in the Supplementary material. Additionally, we ran the comparisons of locus coeruleus CNR between the patients and controls using a more conservative $25 \%$ probability mask and the results were qualitatively identical; this analysis is reported in the Supplementary material.

\section{Stop-signal task performance}

In keeping with the tracking algorithm, the stop accuracy for the Parkinson's disease group on placebo (Fig. $3 \mathrm{~A}$; mean $=0.48, \mathrm{SD}=$ 0.15 ) was not significantly different from controls [mean $=0.58, \mathrm{SD}$ $=0.18 ; \mathrm{t}(40.79)=1.90, P=0.065, \mathrm{BF}=0.35]$. Across the Parkinson's disease patients, group-wise stop accuracy on atomoxetine (mean $=0.45, \mathrm{SD}=0.13$ ) was not significantly different from the placebo session $[t(16)=0.88, P=0.39, \mathrm{BF}=0.35]$. The go error rate approached zero for most participants, yielding a skewed distribution bounded at zero (Fig. 3D), and was therefore logit transformed prior to analysis. ${ }^{84}$ The logit go error rate was slightly higher in the Parkinson's disease group on placebo (mean $=-4.29, \mathrm{SD}=1.23$ ) than in controls $[$ mean $=-5.04, \mathrm{SD}=0.93 ; \mathrm{t}(29.94)=-2.19, \mathrm{P}=$ $0.037, \mathrm{BF}=0.46$ ]. Within the Parkinson's disease group, the logit go error rate was marginally reduced on atomoxetine (mean $=-4.75$, $\mathrm{SD}=1.22)$ compared to placebo $[\mathrm{t}(16)=2.23, \mathrm{P}=0.041, \mathrm{BF}=1.73]$. However, we note that the BF for both these tests fell below conventional thresholds for positive evidence (i.e. $\mathrm{BF}>3$ ), and these effects on go error rate should therefore be regarded as 'anecdotal' at the group level.

\section{Ex-Gaussian model estimates of stop-signal reaction time}

The hierarchical Bayesian estimates of the ex-Gaussian finish time distributions for the stop and matching go processes are shown in Fig. 3B and E, respectively. The stop process finish times tended to be faster for the control group than the Parkinson's disease group. Indeed, the posterior distribution of group-level mean SSRT (Fig. 3C) was lower for the control group (median $=0.39 \mathrm{~s}$, 95\% QI: $0.36,0.41)$ than the Parkinson's disease group on placebo (median $=0.46 \mathrm{~s}, 95 \%$ QI: $0.41,0.52$ ), and this group difference in SSRT was reliably different from zero ( $\Delta$ group median $=0.07 \mathrm{~s}, 95 \%$ QI: 0.01, 0.14). The mean SSRT for the Parkinson's disease group on atomoxetine (median $=0.47 \mathrm{~s}, 95 \%$ QI: $0.42,0.52$ ) was comparable to the placebo session ( $\Delta$ drug median $=0.01 \mathrm{~s}, 95 \%$ QI: $-0.07,0.09$ ).

For the matching go process, the distributions of finish times varied widely across participants, but the group-level distributions were highly similar. The posterior distributions of grouplevel mean go eraction time (Fig. 3F) did not differ between the 

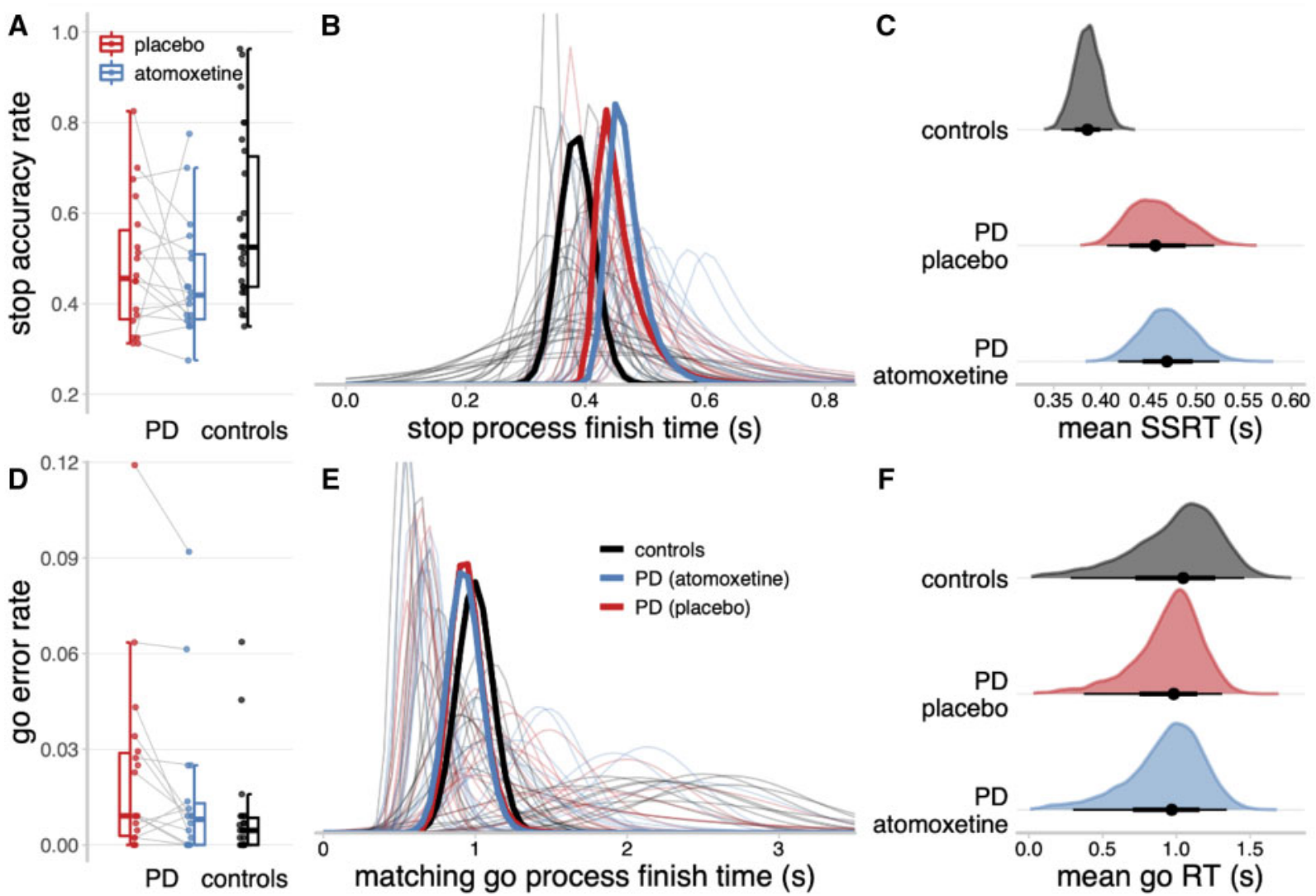

Figure 3 Descriptive statistics and ex-Gaussian model estimates of stop-signal task performance. (A and D) Proportions of successful stop trials (A) and incorrect go responses (D). (B and E) Ex-Gaussian finish time distributions of the stop process (B) and matching go process (E). Bold lines represent group-level mean distributions; thin lines represent individual participants. The mean of a given ex-Gaussian distribution was taken as the SSRT (stop process) or go reaction time (RT) (matching go process). (C and F) Posterior distributions of group-level mean SSRT (C) and go reaction time (F). The black dots represent the medians; the thick black line segments represent the $66 \%$ quantile intervals; and the thin black line segments represent the $95 \%$ quantile intervals. PD = Parkinson's disease.

control group (median $=1.05 \mathrm{~s}, 95 \%$ QI: $0.28,1.46)$, Parkinson's disease group on placebo (median $=0.98 \mathrm{~s}, 95 \%$ QI: 0.37, $1.31 ; \Delta$ group median $=-0.07 \mathrm{~s}, 95 \%$ QI: $-0.78,0.77)$, and Parkinson's disease group on atomoxetine (median $=0.97 \mathrm{~s}, 95 \%$ QI: $0.30,1.34 ; \Delta$ drug median $=-0.01 \mathrm{~s}, 95 \%$ QI: $-0.72,0.67)$. There were also no mean differences between groups or drug conditions for the attentional failure parameters, trigger failure and go failure (Supplementary Fig. 11).

\section{Locus coeruleus integrity and atomoxetine-induced changes in stop-signal reaction time}

Although there was no group-wise effect of atomoxetine on the Parkinson's disease group in terms of their mean SSRT, we predicted that the effect of atomoxetine would depend on individual differences in locus coeruleus integrity, as indexed by the CNR. We confirmed a significant interaction effect between the drug condition and locus coeruleus CNR on the participant-level estimates of SSRT [Fig. $4 \mathrm{~A} ; \beta=0.27, F(1,14.61)=14.61, P=0.002 ; \mathrm{BF}=11.56]$. This interaction effect did not meaningfully change when including clinical covariates such as age, disease severity, atomoxetine plasma level and dopaminergic medication, as both frequentist and Bayesian model selection procedures indicated that such covariates did not significantly improve the model fit (for details see the Supplementary material). We performed several follow-up analyses that confirmed this interaction was not driven by overly influential outlier participants, including Cook's distance, ${ }^{85,86}$
DFBETA $^{87}$ and refitting using a robust estimation method (for details see the Supplementary material). We also showed that the Drug $\times$ Locus coeruleus CNR interaction did not change when additional brain imaging metrics were included as covariates. These metrics included total intracranial volume and CNR extracted from the substantia nigra (details can be found in the Supplementary material). Finally, we found that the interaction did not change when using an alternative calculation for locus coeruleus contrast (contrast ratio to mean, not SD, see Supplementary material), or when the contrast values were obtained using the more conservative $25 \%$ probability mask (Supplementary material).

There was also a main effect of session $[\beta=0.25$, $F(1,14.53)=13.33, P=0.002 ; B F=2.58]$, reflecting slightly shorter SSRTs for the second session compared to the first, regardless of the drug condition. There were no significant main effects of drug condition $[\beta=-0.05, F(1,14.21)=0.70, P=0.416 ; B F=0.37]$ or locus coeruleus CNR $[\beta=0.11, F(1,14.61)=0.266, P=0.613 ; B F=0.55]$ on SSRT.

To understand the Drug $\times$ Locus coeruleus CNR interaction further, we examined the relationship between locus coeruleus CNR and the drug-induced change in SSRT ( $\Delta$ drug: atomoxetine - placebo), adjusted for the main effect of session. This relationship was strongly positive, suggesting that patients with lower locus coeruleus CNR have a greater reduction in SSRT after atomoxetine [Fig. 4B; $r(15)=0.73, P<0.001 ; \mathrm{BF}=32.70$ ].

There was no interaction effect between drug condition and locus coeruleus CNR on the participant-level estimates of go 
A

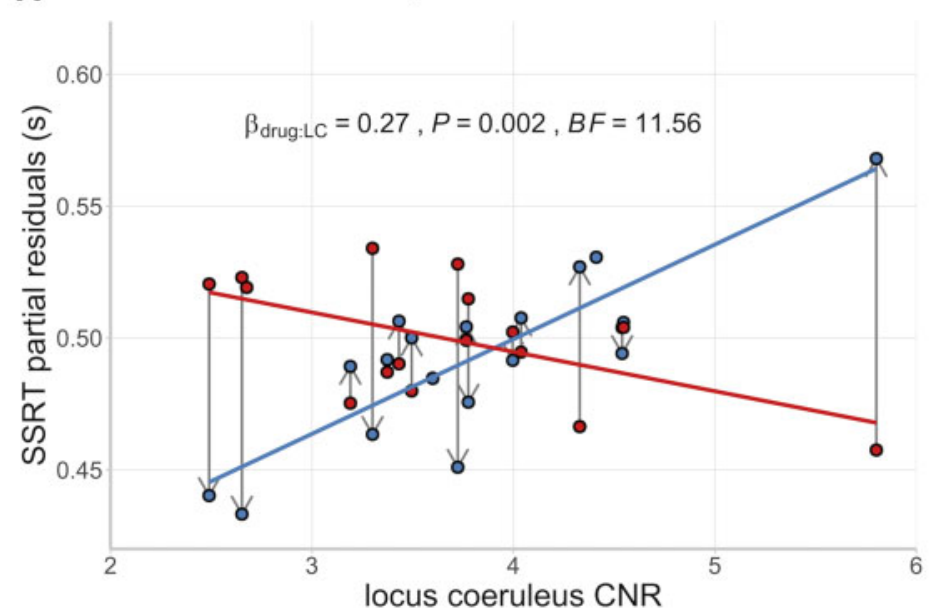

B

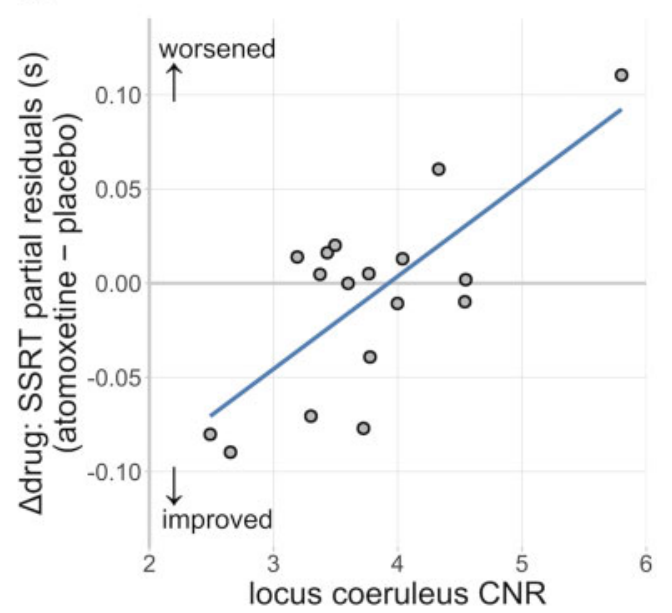

Figure 4 Relationship between SSRT, locus coeruleus and drug. (A) SSRT estimates as a function of drug condition and locus coeruleus CNR. Withinsubject change in SSRT from placebo to atomoxetine is illustrated with vertical grey arrows. (B) Relationship between locus coeruleus CNR and the drug-induced change in SSRT. For visualization purposes, the SSRT estimates were adjusted for the fixed effect of session and random effect of participants (i.e. partial residuals).

reaction time $[\beta=0.01, F(1,16.37)=0.00, P=0.971 ; B F=0.47]$, stop accuracy rate $[\beta=-0.18, F(1,16.52)=1.15, P=0.300 ; B F=0.58]$, or logit go error rate $[\beta=-0.02, F(1,15.14)=0.03, P=0.865 ; B F=0.46]$. Finally, to explore the possibility of hemisphere-specific effects, we ran the linear mixed model analysis separately for CNR values from the left and right locus coeruleus. These results were qualitatively identical to the original combined analysis, arguing against hemisphere-specific effects (Supplementary material).

\section{Discussion}

We show that improvements in response inhibition after atomoxetine are dependent on locus coeruleus integrity in patients with Parkinson's disease. Following a single $40 \mathrm{mg}$ dose of atomoxetine, individuals with lower locus coeruleus integrity had a greater improvement in response inhibition (i.e. reduction in their SSRT). This result highlights the link between integrity of the noradrenergic locus coeruleus and action cancellation, which has previously been inferred from preclinical work and pharmacological manipulations. The finding also demonstrates the importance of baseline noradrenergic capacity in determining the response to atomoxetine, confirming the need to stratify patients for noradrenergic therapy. Locus coeruleus neuromelanin imaging would be a safe and affordable means to achieve this stratification.

Previous work in Parkinson's disease showed that atomoxetine led to greater improvements in SSRT and enhanced activation in the stopping network in patients with more severe disease. ${ }^{11,12}$ Extending this work, we confirm that improved SSRTs under atomoxetine occurred in those patients with more severe locus coeruleus degeneration. This was in the context of a general impairment in response inhibition, as patients on placebo had longer SSRTs compared to controls. Our result suggests that a single $40 \mathrm{mg}$ atomoxetine dose confers the most benefit on individuals with a severe loss of noradrenergic capacity. In this way, noradrenergic replacement in patients with a compromised system may achieve restoration closer to normal levels and improve behaviour. Conversely, in patients with a less affected system the same dose may offer no appreciable benefit or even 'overdose' the system, leading to worse behaviour. This relationship is captured by the inverted U-shaped curve (known as a Yerkes-Dodson function) that is common across monoaminergic and cholinergic systems, whereby intermediate levels of neuromodulatory influence are associated with optimal performance, with too much or too little having deleterious effects on behaviour. $^{25,88}$

Baseline dependency in dose-response curves is well documented in relation to dopaminergic therapy, ${ }^{89,90}$ such that the optimal level of dopamine enhancement needed to improve behaviour depends on pre-existing dopamine levels. This has had critical implications for Parkinson's disease therapy. Dopamine dosages titrated to restore levels in the severely depleted dorsal striatum and motor system circuitry effectively overdose the less affected ventral tegmental area, ventral striatum and associated limbic pathways, ${ }^{91}$ impairing aspects of learning and cognitive flexibility. ${ }^{92-94}$ Our result suggests a similar baseline dependency for noradrenergic therapy, where optimal dosages needed for atomoxetine therapy may depend on the extent of degeneration in the locus coeruleus. This has important implications for optimizing noradrenergic therapy in Parkinson's disease, as patients could be stratified based on locus coeruleus integrity to inform appropriate dosages in clinical trials or personalized treatment. ${ }^{41}$

Neuromelanin-sensitive MT imaging of the locus coeruleus represents a promising avenue to achieve this stratification. Although we have previously shown a relationship between disease severity (as measured by the UPDRS-III) and atomoxetine responsivity, such measures of motor function or disease duration may not be the most accurate measure of noradrenergic capacity. Whilst progressive degeneration of the locus coeruleus is expected over the disease course, this will vary widely across individuals and will reflect the disease phenotype. Neuropathological studies and neuromelanin imaging have shown more pronounced locus coeruleus degeneration in certain phenotypes, including those with cognitive impairment or dementia, ${ }^{95-97}$ depression, ${ }^{98}$ an akinetic-rigid syndrome ${ }^{99}$ and REM sleep behaviour disorder, ${ }^{100,101}$ relative to patients at equivalent disease stages. In our results we note that including the MDS-UPDRS-III as an index of disease severity did not meaningfully improve the model fit for the interaction between the drug condition and locus coeruleus CNR. This 
highlights the added value of locus coeruleus imaging, above and beyond disease severity metrics, to explain variations in atomoxetine responsivity.

Our locus coeruleus imaging identified that the greatest difference between controls and patients was in the caudal portion of the locus coeruleus. This has not previously been identified in Parkinson's disease locus coeruleus imaging with $3 \mathrm{~T}$ MRI, which limited analysis to the whole structure. This is because of the inherent limitations in 3 T MRI, where voxel sizes and slice thickness are larger and do not allow for the spatial resolution needed to examine locus coeruleus subdivisions. ${ }^{45}$ While some neuropathology studies have noted comparable cell loss across the rostral-caudal extent of the locus coeruleus, ${ }^{102,103}$ others have reported more severe degenerative changes in the caudal segment. ${ }^{104}$ Previous studies have validated the relationship between neuromelaninsensitive MRI signal intensity and histological measures of the density of neuromelanin-accumulating neurons ${ }^{105-107}$ and neuromelanin concentration. ${ }^{108}$ In light of these links, neuromelaninsensitive MRI is considered a promising biomarker of Parkinson disease-related neurodegenerative processes. ${ }^{109}$

Our study aimed for noradrenergic modulation of the SSRT. Atomoxetine increases extracellular noradrenaline levels across the brain via its actions at the noradrenaline transporter, in particular increasing levels in the prefrontal cortex by 3 -fold. ${ }^{32,110}$ However, atomoxetine may also increase extracellular dopamine levels, particularly in prefrontal cortex..$^{32}$ Because of the relative sparsity of dopamine transporters in the prefrontal cortex, ${ }^{111} \mathrm{a}$ portion of dopamine uptake is mediated by the noradrenaline transporter. ${ }^{112,113}$ In this way, improved response inhibition under atomoxetine in Parkinson's disease might potentially also reflect elevated prefrontal dopamine levels. The respective roles of noradrenergic versus dopaminergic modulation over the SSRT is not fully resolved. Some studies in rodents and humans have implicated a selective link between noradrenergic transmission and action cancellation: increasing dopamine selectively does not affect the SSRT. ${ }^{114-117}$ This work has been taken in concert with studies using agents that non-selectively change both dopamine and noradrenaline and do affect the SSRT. For example, haloperidol and methylphenidate are shown to modulate the SSRT, having both dopaminergic and non-dopaminergic effects. ${ }^{118-121}$ One interpretation of this body of work is that the effects of the non-selective drugs are primarily driven by their noradrenergic actions, given that selective dopamine manipulations do not reliably modulate SSRT. However, some studies have found modulation of the SSRT using relatively selective dopamine drugs ${ }^{122,123}$ and dopamine receptor availability is associated with SSRT performance. ${ }^{124}$ The specificity of noradrenergic versus dopaminergic modulation of the SSRT is not fully elucidated. These two closely related neurotransmitters share a common biosynthetic pathway. They can be co-released from the same terminals ${ }^{125-127}$ and both modulate goal-directed behaviour. ${ }^{128}$ Although we propose that the principal effect of atomoxetine on SSRT is mediated via noradrenergic mechanisms, dopaminergic effects may also contribute.

In practical terms, a key question is whether 'noradrenergic' drugs offer a useful strategy to treat inhibitory control deficits. The association we have shown between locus coeruleus integrity and the change in SSRT under atomoxetine suggests a causal association between the noradrenergic system and action cancellation in Parkinson's disease. Our post hoc supplementary analyses with brain volume and substantia nigra integrity showed these covariates did not influence that association. This indicates a degree of specificity for the locus coeruleus-noradrenergic system's role in the SSRT in Parkinson's disease. However, future cross-over studies using specific noradrenergic versus dopaminergic agents would be beneficial to resolve residual ambiguity. Our current results point towards atomoxetine as a useful strategy to treat inhibitory deficits in Parkinson's disease, in the context of appropriate stratification using locus coeruleus integrity.

Our results do not speak directly to atomoxetine's mechanism of action in Parkinson's disease. However, convergent evidence indicates that atomoxetine may increase efficiency in brain networks mediating response inhibition, via actions at the prefrontal cortex and the locus coeruleus. ${ }^{32,40}$ In patients with Parkinson's disease, atomoxetine has been shown to increase activity within and between regions of the stopping network, including the presupplementary motor area and inferior frontal gyrus. ${ }^{11,12}$ Locus coeruleus degeneration in Parkinson's disease is accompanied by reduced noradrenaline levels in forebrain regions. ${ }^{95,129,130}$ As noradrenaline release facilitates reconfigurations of large-scale networks, ${ }^{26,27}$ depletion of forebrain noradrenaline is likely to impact the rapid engagement of brain network activity that is necessary for successful action cancellation. ${ }^{131}$ In patients with greater locus coeruleus degeneration, which may be accompanied by decreased or dysfunctional modulation of prefrontal noradrenergic targets, efficiency of the stopping network may be reduced. Consequently, these patients show the greatest benefit from a drug that can increase levels of prefrontal noradrenaline and upregulate locus coeruleus function.

We would support the use of $40 \mathrm{mg}$ atomoxetine in subsequent single-dose studies in Parkinson's disease. Based on the current results, as well as previous clinical trials ${ }^{53,54}$ and mechanistic studies, ${ }^{10-12}$ this dose is very well tolerated and capable of modulating neural systems and behaviour. Future clinical trials may consider a dose-ranging algorithm based on tolerability, plasma levels or behaviour, with the potential to increase beyond a $40 \mathrm{mg}$ starter dose. $22,53,54$ Gradually increasing the dose over the course of treatment is consistent with animal models of atomoxetine, which confirm that increased levels of prefrontal extracellular noradrenaline following an acute dose are sustained over chronic administration, but not to the same extent. ${ }^{132}$ Increasing the dose over the course of treatment may be necessary to maintain equivalent behavioural benefits. Future clinical studies may also consider doses based on genetic stratification of patients, specifically, allelic variation in the noradrenaline transporter gene (SLC6A2) and the CYP2D6 enzyme gene. These are known to modulate clinical response to atomoxetine $^{133-135}$ and absorption, bioavailability and half-life. ${ }^{55}$ Our results suggest that locus coeruleus imaging may also serve as a cost-effective means of stratifying patients' atomoxetine dose in future clinical settings, mitigating the need for ongoing visits to titrate doses.

Our study was designed to test a mechanistic hypothesis around locus coeruleus integrity and atomoxetine responsivity; therefore, we did not focus on clinically relevant outcomes. Future longer-term clinical trials are needed to determine if atomoxetine improves cognition and behaviour in a way that benefits daily function, including carer and patient ratings. The link between response inhibition and atomoxetine suggests that an important phenotype to focus on will be those patients with impulsivity, even where this falls short of an impulse control disorder. We also note that locus coeruleus degeneration has been strongly linked with REM sleep behaviour disorder. ${ }^{100,101}$ Future studies could selectively recruit those patients with impulse control disorders and/ or polysomnography-confirmed REM sleep behaviour disorder in order to establish the clinical benefits of atomoxetine in these stratified phenotypes.

Given the role of noradrenaline in cognition and behaviour, ${ }^{136}$ noradrenergic dysfunction contributes to cognitive deficits beyond action cancellation in Parkinson's disease. Optimizing 
noradrenergic therapy therefore has potential to provide relief across a variety of non-motor symptoms. ${ }^{137}$ Our results confirm the potential for stratified noradrenergic therapy in Parkinson's disease, whereby the efficacy of these drugs varies across individuals depending on their baseline noradrenergic state. Locus coeruleus neuromelanin imaging may offer a marker of noradrenergic capacity that can be used to stratify patients to optimise successful outcomes in trials of noradrenergic therapy, and ultimately inform a more personalized treatment approach.

\section{Acknowledgements}

We thank all volunteers and their families for their participation, all staff at the Wolfson Brain Imaging Centre and NIHR Cambridge Clinical Research Facility for their help with data collection, and members of the Cambridge Centre for Frontotemporal Dementia and Related Disorders for valuable suggestions and discussions.

\section{Funding}

This study was supported by Parkinson's UK (grant number K1702) and the Cambridge Centre for Parkinson-plus. C.O. was supported by a Neil Hamilton Fairley Fellowship from the Australian National Health and Medical Research Council (GNT1091310). F.H.H. was supported by a Cambridge Trust Vice-Chancellor's Award and Fitzwilliam College Scholarship. N.H. was supported by the Association of British Neurologists-Patrick Berthoud Charitable Trust (RG99368). K.A.T. was supported by supported by the British Academy (PF160048) and the Guarantors of Brain (101149). A.G.M. was supported by the Holt Fellowship (RG86564). C.H.W.-G. is supported by a RCUK/UKRI Research Innovation Fellowship awarded by the Medical Research Council (MR/R007446/1). J.B.R. was supported by a UK Medical Research Council Intramural Programme Grant (SUAG/051 G101400) and Research Grant (MR/P01271X/1), a James S. McDonnell Foundation 21st Century Science Initiative Scholar Award in Understanding Human Cognition, and the Wellcome Trust (220258). This study was carried out at/supported by the NIHR Cambridge Clinical Research Facility and the NIHR Cambridge Biomedical Research Centre Dementia and Neurodegeneration Theme (ref. 146281) The views expressed are those of the author(s) and not necessarily those of the NHS, the NIHR or the Department of Health and Social Care.

\section{Competing interests}

The authors report no competing interests.

\section{Supplementary material}

Supplementary material is available at Brain online.

\section{References}

1. Cools R, Barker RA, Sahakian BJ, Robbins TW. l-Dopa medication remediates cognitive inflexibility, but increases impulsivity in patients with Parkinson's disease. Neuropsychol. 2003; 41(11):1431-1441.

2. Lewis SJG, Slabosz A, Robbins TW, Barker RA, Owen AM. Dopaminergic basis for deficits in working memory but not attentional set-shifting in Parkinson's disease. Neuropsychol. 2005;43(6):823-832.

3. Kehagia AA, Barker RA, Robbins TW. Neuropsychological and clinical heterogeneity of cognitive impairment and dementia in patients with Parkinson's disease. Lancet Neurol. 2010;9(12): 1200-1213.

4. Halliday GM, Leverenz JB, Schneider JS, Adler CH. The neurobiological basis of cognitive impairment in Parkinson's disease. Mou Disord. 2014;29(5):634-650.

5. Braak H, Tredici KD, Rüb U, de Vos RAI, Jansen Steur ENH, Braak E. Staging of brain pathology related to sporadic Parkinson's disease. Neurobiol Aging. 2003;24(2):197-211.

6. Surmeier DJ, Obeso JA, Halliday GM. Selective neuronal vulnerability in Parkinson disease. Nat Rev Neurosci. 2017;18(2):101-113.

7. Weinshenker $D$. Long road to ruin: Noradrenergic dysfunction in neurodegenerative disease. Trends Neurosci. 2018;41(4): 211-223.

8. Eagle DM, Bari A, Robbins TW. The neuropsychopharmacology of action inhibition: Cross-species translation of the stop-signal and go/no-go tasks. Psychopharmacology (Berl). 2008;199(3): 439-456.

9. Vazey EM, Aston-Jones G. The emerging role of norepinephrine in cognitive dysfunctions of Parkinson's disease. Front Behav Neurosci. 2012;6:48.

10. Kehagia AA, Housden CR, Regenthal R, et al. Targeting impulsivity in Parkinson's disease using atomoxetine. Brain. 2014; 137(Pt 7):1986-1997.

11. Ye Z, Altena E, Nombela C, et al. Improving response inhibition in Parkinson's disease with atomoxetine. Biol Psychiatry. 2015; 77(8):740-748.

12. Rae CL, Nombela C, Rodríguez PV, et al. Atomoxetine restores the response inhibition network in Parkinson's disease. Brain. 2016;139(Pt 8):2235-2248.

13. Gauggel S, Rieger M, Feghoff T-A. Inhibition of ongoing responses in patients with Parkinson's disease. J Neurol Neurosurg Psychiatry. 2004;75(4):539-544.

14. Obeso I, Wilkinson L, Casabona E, et al. Deficits in inhibitory control and conflict resolution on cognitive and motor tasks in Parkinson's disease. Exp Brain Res. 2011;212(3):371-384.

15. O'Callaghan C, Naismith SL, Hodges JR, Lewis SJG, Hornberger M. Fronto-striatal atrophy correlates of inhibitory dysfunction in Parkinson's disease versus behavioural variant frontotemporal dementia. Cortex. 2013;49(7):1833-1843.

16. Nombela C, Rittman T, Robbins TW, Rowe JB. Multiple modes of impulsivity in Parkinson's disease. PLoS One. 2014;9(1):e85747.

17. Napier TC, Corvol J-C, Grace AA, et al. Linking neuroscience with modern concepts of impulse control disorders in Parkinson's disease. Mou Disord. 2015;30(2):141-149.

18. Weintraub D, Koester J, Potenza MN, et al. Impulse control disorders in Parkinson disease: A cross-sectional study of 3090 patients. Arch Neurol. 2010;67(5):589-595.

19. O'Callaghan C, Bertoux M, Hornberger M. Beyond and below the cortex: The contribution of striatal dysfunction to cognition and behaviour in neurodegeneration. J Neurol Neurosurg Psychiatry. 2014;85(4):371-378.

20. Jahanshahi M, Obeso I, Baunez C, et al. Parkinson's disease, the subthalamic nucleus, inhibition, and impulsivity.Mov Disord. 2015;30(2):128-140.

21. Mosley PE, Paliwal S, Robinson K, et al. The structural connectivity of discrete networks underlies impulsivity and gambling in Parkinson's disease. Brain. 2019;142(12):3917-3935.

22. Marsh L, Biglan K, Gerstenhaber M, Williams JR. Atomoxetine for the treatment of executive dysfunction in Parkinson's disease: A pilot open-label study. Mou Disord. 2009;24(2):277-282.

23. Robbins TW, Arnsten AFT. The Neuropsychopharmacology of fronto-executive function: Monoaminergic modulation. Annu Rev Neurosci. 2009;32(1):267-287.

24. Bari A, Robbins TW. Inhibition and impulsivity: Behavioral and neural basis of response control. Prog Neurobiol. 2013;108:44-79. 
25. Aston-Jones G, Cohen JD. An integrative theory of locus coeruleus-norepinephrine function: Adaptive gain and optimal performance. Annu Rev Neurosci. 2005;28(1):403-450.

26. Bouret S, Sara SJ. Network reset: A simplified overarching theory of locus coeruleus noradrenaline function. Trends Neurosci. 2005;28(11):574-582.

27. Zerbi V, Floriou-Servou A, Markicevic M, et al. Rapid reconfiguration of the functional connectome after chemogenetic locus coeruleus activation. Neuron. 2019;103(4):702-718.e5.

28. Chamberlain SR, Müller U, Blackwell AD, Clark L, Robbins TW, Sahakian BJ. Neurochemical modulation of response inhibition and probabilistic learning in humans. Science. 2006; 311(5762):861-863.

29. Robinson ESJ, Eagle DM, Mar AC, et al. Similar effects of the selective noradrenaline reuptake inhibitor atomoxetine on three distinct forms of impulsivity in the rat. Neuropsychopharmacol. 2008;33(5):1028-1037.

30. Chamberlain SR, Hampshire A, Müller U, et al. Atomoxetine modulates right inferior frontal activation during inhibitory control: A pharmacological functional magnetic resonance imaging study. Biol Psychiatry. 2009;65(7):550-555.

31. Bari A, Mar AC, Theobald DE, et al. Prefrontal and monoaminergic contributions to stop-signal task performance in rats. $J$ Neurosci. 2011;31(25):9254-9263.

32. Bymaster FP, Katner JS, Nelson DL, et al. Atomoxetine increases extracellular levels of norepinephrine and dopamine in prefrontal cortex of rat: A potential mechanism for efficacy in attention deficit/hyperactivity disorder. Neuropsychopharmacol. 2002;27(5):699-711.

33. Aron AR. From reactive to proactive and selective control: Developing a richer model for stopping inappropriate responses. Biol Psychiatry. 2011;69(12):e55-e68.

34. Rae CL, Hughes LE, Weaver C, Anderson MC, Rowe JB. Selection and stopping in voluntary action: A meta-analysis and combined fMRI study. Neuroimage. 2014;86:381-391.

35. McGinley MJ, David SV, McCormick DA. Cortical membrane potential signature of optimal states for sensory signal detection. Neuron. 2015;87(1):179-192.

36. Eldar E, Cohen JD, Niv Y. The effects of neural gain on attention and learning. Nat Publ Group. 2013;16(8):1146-1153.

37. Shine JM, van den Brink RL, Hernaus D, Nieuwenhuis S, Poldrack RA. Catecholaminergic manipulation alters dynamic network topology across cognitive states. Netw Neurosci. 2018; 2(3):381-396.

38. Arnsten AFT, Goldman-Rakic PS. Selective prefrontal cortical projections to the region of the locus coeruleus and raphe nuclei in the rhesus monkey. Brain Res. 1984;306(1-2):9-18.

39. Jodo E, Chiang C, Aston-Jones G. Potent excitatory influence of prefrontal cortex activity on noradrenergic locus coeruleus neurons. Neuroscience. 1998;83(1):63-79.

40. Bari A, Aston-Jones G. Atomoxetine modulates spontaneous and sensory-evoked discharge of locus coeruleus noradrenergic neurons. Neuropharmacol. 2013;64(c):53-64.

41. Ye Z, Rae CL, Nombela C, et al. Predicting beneficial effects of atomoxetine and citalopram on response inhibition in Parkinson's disease with clinical and neuroimaging measures: Predicting Treatment Response in PD. Hum Brain Mapp. 2016; 37(3):1026-1037.

42. Matthews PM, Edison P, Geraghty OC, Johnson MR. The emerging agenda of stratified medicine in neurology. Nat Rev Neurol. 2014;10(1):15-26.

43. Priovoulos N, Jacobs HIL, Ivanov D, Uludağ K, Verhey FRJ, Poser BA. High-resolution in vivo imaging of human locus coeruleus by magnetization transfer MRI at 3T and 7T. Neuroimage. 2018; 168:427-436.
44. Betts MJ, Kirilina E, Otaduy MCG, et al. Locus coeruleus imaging as a biomarker for noradrenergic dysfunction in neurodegenerative diseases. Brain. 2019;142(9):2558-2514.

45. Ye R, Rua C, O'Callaghan C, et al. An in vivo probabilistic atlas of the human locus coeruleus at ultra-high field. Neuroimage. 2021;225:117487.

46. Emre M, Aarsland D, Brown R, et al. Clinical diagnostic criteria for dementia associated with Parkinson's disease. Mou Disord. 2007;22(12):1689-1707.

47. Martinez-Martin P, Falup-Pecurariu C, Rodriguez-Blazquez C, et al. Dementia associated with Parkinson's disease: Applying the movement disorder society task force criteria. Parkinsonism Relat Disord. 2011;17(8):621-624.

48. Weintraub D, Hoops S, Shea JA, et al. Validation of the questionnaire for impulsive-compulsive disorders in Parkinson's disease. Mov Disord. 2009;24(10):1461-1467.

49. Tomlinson CL, Stowe R, Patel S, Rick C, Gray R, Clarke CE. Systematic review of levodopa dose equivalency reporting in Parkinson's disease. Mov Disord. 2010;25(15):2649-2653.

50. Goetz CG, Tilley BC, Shaftman SR, et al.; Movement Disorder Society UPDRS Revision Task Force. Movement Disorder Society-sponsored revision of the Unified Parkinson's Disease Rating Scale (MDS-UPDRS): Scale presentation and clinimetric testing results. Mov Disord. 2008;23(15):2129-2170.

51. Nasreddine ZS, Phillips NA, Bédirian V, et al. The Montreal Cognitive Assessment, MoCA: A brief screening tool for mild cognitive impairment. J Am Geriatr Soc. 2005;53(4):695-699.

52. Mioshi E, Dawson K, Mitchell J, Arnold R, Hodges JR. The Addenbrooke's Cognitive Examination Revised (ACE-R): A brief cognitive test battery for dementia screening. Int $J$ Geriatr Psychiatry. 2006;21(11):1078-1085.

53. Weintraub D, Mavandadi S, Mamikonyan E, et al. Atomoxetine for depression and other neuropsychiatric symptoms in Parkinson disease. Neurology. 2010;75(5):448-455.

54. Hinson VK, Delambo A, Elm J, Turner T. A randomized clinical trial of atomoxetine for mild cognitive impairment in Parkinson's disease. Mou Disord Clin Pract. 2017;4(3): 416-423.

55. Sauer J-M, Ring BJ, Witcher JW. Clinical pharmacokinetics of atomoxetine. Clin Pharmacokinet. 2005;44(6):571-590.

56. Teichert J, Rowe JB, Ersche KD, et al. Determination of atomoxetine or escitalopram in human plasma by HPLC: Applications in neuroscience research studies. Int $J$ Clin Pharmacol Ther. 2020;58(08):426-438.

57. Heathcote A, Lin Y-S, Reynolds A, Strickland L, Gretton M, Matzke D. Dynamic models of choice. Behav Res Methods. 2019; 51(2):961-985.

58. Verbruggen F, Aron AR, Band GP, et al. A consensus guide to capturing the ability to inhibit actions and impulsive behaviors in the stop-signal task. eLife. 2019;8:e55-e26.

59. Matzke D, Dolan CV, Logan GD, Brown SD, Wagenmakers E-J. Bayesian parametric estimation of stop-signal reaction time distributions. J Exp Psychol Gen. 2013;142(4):1047-1073.

60. Ratcliff R. Group reaction time distributions and an analysis of distribution statistics. Psychol Bull. 1979;86(3):446-461.

61. Heathcote A, Popiel SJ, Mewhort DJ. Analysis of response time distributions: An example using the Stroop task. Psychol Bull. 1991;109(2):340-347.

62. Matzke D, Curley S, Gong CQ Heathcote A. Inhibiting responses to difficult choices. J Exp Psychol Gen. 2019;148(1):124-142.

63. Matzke D, Love J, Heathcote A. A Bayesian approach for estimating the probability of trigger failures in the stop-signal paradigm. 2017; Behav Res Methods. 2017;49(1):267-281.

64. Skippen P, Matzke D, Heathcote A, Fulham WR, Michie P, Karayanidis F. Reliability of triggering inhibitory process is a 
better predictor of impulsivity than SSRT. Acta Psychol (Amst). 2019;192:104-117.

65. Matzke D, Hughes M, Badcock JC, Michie P, Heathcote A. Failures of cognitive control or attention? The case of stop-signal deficits in schizophrenia. Atten Percept Psychophys. 2017; 79(4):1078-1086.

66. Weigard A, Heathcote A, Matzke D, Huang-Pollock C. Cognitive modeling suggests that attentional failures drive longer stopsignal reaction time estimates in attention deficit/hyperactivity disorder. Clin Psychol Sci. 2019;7(4):856-872.

67. Band GPH, van der Molen MW, Logan GD. Horse-race model simulations of the stop-signal procedure. Acta Psychol (Amst). 2003;112(2):105-142.

68. Kruschke JK. Bayesian estimation supersedes the t test. J Exp Psychol Gen. 2013;142(2):573-603.

69. Kass RE, Raftery AE. Bayes factors. J Am Stat Assoc. 1995; 90(430):773-795.

70. Rouder JN, Speckman PL, Sun D, Morey RD, Iverson G. Bayesian $t$ tests for accepting and rejecting the null hypothesis. Psychon Bull Rev. 2009;16(2):225-237.

71. Rouder JN, Morey RD, Speckman PL, Province JM. Default Bayes factors for ANOVA designs. J Math Psychol. 2012;56(5):356-374.

72. Hinne M, Gronau QF, van den Bergh D, Wagenmakers E-J. A Conceptual Introduction to Bayesian Model Averaging. Adv Methods Pract Psychol Sci. 2020;3(2):200-215.

73. Kleiner M, Brainard D, Pelli D. What's new in Psychtoolbox-3? Perception. 2007;36(Suppl):14.

74. Wickham H, Averick M, Bryan J, et al. Welcome to the Tidyverse. J Open Source Softw. 2019;4(43):1686.

75. Kay M. tidybayes: Tidy data and geoms for Bayesian models. Version 2.0.3. Zenodo. 2020. doi:10.5281/zenodo. 3740308

76. Singmann H, Bolker B, Westfall J, et al. Afex: Analysis of factorial experiments. 2020. Accessed 18 June 2020. https://CRAN.R-pro ject.org/package $=$ afex

77. Lenth R, Singmann H, Love J, Buerkner P, Herve M. Emmeans: Estimated Marginal Means, Aka Least-Squares Means. Accessed 10 July 2020. https://CRAN.R-project.org/package=emmeans

78. Morey RD, Rouder JN, Jamil T, Urbanek S, Forner K, Ly A. BayesFactor: Computation of Bayes factors for common designs. Accessed 18 June 2020. https://CRAN.R-project.org/pack age $=$ BayesFactor

79. Makowski D, Ben-Shachar MS, Lüdecke D. bayestestR: Describing effects and their uncertainty, existence and significance within the Bayesian framework. J Open Source Softw. 2019;4(40):1541.

80. Clarke WT, Mougin O, Driver ID, et al. Multi-site harmonization of 7 tesla MRI neuroimaging protocols. Neuroimage. 2020; 206:116335.

81. Tustison NJ, Avants BB, Cook PA, et al. N4ITK: Improved N3 bias correction. IEEE Trans Med Imaging. 2010;29(6):1310-1320.

82. Fonov V, Evans AC, Botteron K, Almli CR, McKinstry RC, Collins DL; Brain Development Cooperative Group. Unbiased average age-appropriate atlases for pediatric studies. Neuroimage. 2011; 54(1):313-327.

83. Avants BB, Epstein CL, Grossman M, Gee JC. Symmetric diffeomorphic image registration with cross-correlation: Evaluating automated labeling of elderly and neurodegenerative brain. Med Image Anal. 2008;12(1):26-41.

84. Warton DI, Hui FKC. The arcsine is asinine: The analysis of proportions in ecology. Ecology. 2011;92(1):3-10.

85. Cook RD. Detection of influential observation in linear regression. Technometrics. 1977;19(1):15-18.

86. Snijders TAB, Berkhof J. Diagnostic checks for multilevel models. In: de Leeuw J, Meijer E, eds. Handbook of multilevel analysis. Springer; 2008:141-175.
87. Fox J, Monette G. An R and S-plus companion to applied regression. Sage Publications; 2002.

88. Robbins TW. Chemical neuromodulation of frontal-executive functions in humans and other animals. Exp Brain Res. 2000; 133(1):130-138.

89. Rowe JB, Hughes L, Ghosh BCP, et al. Parkinson's disease and dopaminergic therapy-differential effects on movement, reward and cognition. Brain. 2008;131(Pt 8):2094-2105.

90. Cools R, D'Esposito M. Inverted-U-shaped dopamine actions on human working memory and cognitive control. Biol Psychiatry. 2011;69(12):e113-e125.

91. Cools R, Barker RA, Sahakian BJ, Robbins TW. Enhanced or impaired cognitive function in Parkinson's disease as a function of dopaminergic medication and task demands. Cereb Cortex. 2001;11(12):1136-1143.

92. Cools R, Lewis SJG, Clark L, Barker RA, Robbins TW. L-DOPA disrupts activity in the nucleus accumbens during reversal learning in Parkinson's disease. Neuropsychopharmacol. 2007; 32(1):180-189.

93. Aarts E, Nusselein AAM, Smittenaar P, Helmich RC, Bloem BR, Cools R. Greater striatal responses to medication in Parkinson's disease are associated with better task-switching but worse reward performance. Neuropsychol. 2014;62:390-397.

94. MacDonald PA, MacDonald AA, Seergobin KN, et al. The effect of dopamine therapy on ventral and dorsal striatum-mediated cognition in Parkinson's disease: support from functional MRI. Brain. 2011;134(Pt 5):1447-1463.

95. Cash R, Dennis T, L'Heureux R, Raisman R, Javoy-Agid F, Scatton B. Parkinson's disease and dementia: Norepinephrine and dopamine in locus ceruleus. Neurology. 1987;37(1):42-42.

96. Zweig RM, Cardillo JE, Cohen M, Giere S, Hedreen JC. The locus ceruleus and dementia in Parkinson's disease. Neurology. 1993; 43(5):986-991.

97. Li Y, Wang C, Wang J, et al. Mild cognitive impairment in de novo Parkinson's disease: A neuromelanin MRI study in locus coeruleus. Mov Disord. 2019;34(6):884-892.

98. Wang J, Li Y, Huang Z, et al. Neuromelanin-sensitive magnetic resonance imaging features of the substantia nigra and locus coeruleus in de novo Parkinson's disease and its phenotypes. Eur J Neurol. 2018;25(7):949-973.

99. Paulus $\mathrm{W}$, Jellinger $\mathrm{K}$. The neuropathologic basis of different clinical subgroups of Parkinson's disease. J Neuropathol Exp Neurol. 1991;50(6):743-755.

100. García-Lorenzo D, Longo-Dos Santos C, Ewenczyk C, et al. The coeruleus/subcoeruleus complex in rapid eye movement sleep behaviour disorders in Parkinson's disease. Brain. 2013;136(Pt 7):2120-2129.

101. Sommerauer M, Fedorova TD, Hansen AK, et al. Evaluation of the noradrenergic system in Parkinson's disease: An 11C-MeNER PET and neuromelanin MRI study. Brain. 2018;141(2):496-504.

102. Chan-Palay V, Asan E. Alterations in catecholamine neurons of the locus coeruleus in senile dementia of the Alzheimer type and in Parkinson's disease with and without dementia and depression. J Comp Neurol. 1989;287(3):373-392.

103. German DC, Manaye KF, White CL, et al. Disease-specific patterns of locus coeruleus cell loss. Ann Neurol. 1992;32(5):667-676.

104. Bertrand E, Lechowicz W, Szpak GM, Dymecki J. Qualitative and quantitative analysis of locus coeruleus neurons in Parkinson's disease. Folia Neuropathol. 1997;35(2):80-86.

105. Kitao S, Matsusue E, Fujii S, et al. Correlation between pathology and neuromelanin MR imaging in Parkinson's disease and dementia with Lewy bodies. Neuroradiology. 2013;55(8):947-953.

106. Keren NI, Taheri S, Vazey EM, et al. Histologic validation of locus coeruleus MRI contrast in post-mortem tissue. Neuroimage. 2015;113(C):235-245. 
107. Lee H, Baek S-Y, Kim E-J, Huh GY, Lee J-H, Cho H. MRI T2 and $\mathrm{T} 2$ relaxometry to visualize neuromelanin in the dorsal substantia nigra pars compacta. Neuroimage. 2020;211:116625.

108. Cassidy CM, Zucca FA, Girgis RR, et al. Neuromelanin-sensitive MRI as a noninvasive proxy measure of dopamine function in the human brain. Proc Natl Acad Sci U S A. 2019;116(11): 5108-5117.

109. Sulzer D, Cassidy C, Horga G, et al. Neuromelanin detection by magnetic resonance imaging (MRI) and its promise as a biomarker for Parkinson's disease. NPJ Park Dis. 2018;4:11.

110. Swanson CJ, Perry KW, Koch-Krueger S, Katner J, Svensson KA, Bymaster FP. Effect of the attention deficit/hyperactivity disorder drug atomoxetine on extracellular concentrations of norepinephrine and dopamine in several brain regions of the rat. Neuropharmacol. 2006;50(6):755-760.

111. Sesack SR, Hawrylak VA, Matus C, Guido MA, Levey AI. Dopamine axon varicosities in the prelimbic division of the rat prefrontal cortex exhibit sparse immunoreactivity for the dopamine transporter. J Neurosci. 1998;18(7):2697-2708.

112. Wayment HK, Schenk JO, Sorg BA. Characterization of extracellular dopamine clearance in the medial prefrontal cortex: Role of monoamine uptake and monoamine oxidase inhibition. J Neurosci. 2001;21(1):35-44.

113. Seamans JK, Yang CR. The principal features and mechanisms of dopamine modulation in the prefrontal cortex. Prog Neurobiol. 2004;74(1):1-58.

114. Overtoom CCE, Verbaten MN, Kemner C, et al. Effects of methylphenidate, desipramine, and l-dopa on attention and inhibition in children with Attention Deficit Hyperactivity Disorder. Behav Brain Res. 2003;145(1-2):7-15.

115. Bari A, Eagle DM, Mar AC, Robinson ESJ, Robbins TW. Dissociable effects of noradrenaline, dopamine, and serotonin uptake blockade on stop task performance in rats. Psychopharmacol (Berl.). 2009;205(2):273-283.

116. Obeso I, Wilkinson L, Jahanshahi M. Levodopa medication does not influence motor inhibition or conflict resolution in a conditional stop-signal task in Parkinson's disease. Exp Brain Res. 2011;213(4):435-445.

117. Rawji V, Rocchi L, Foltynie T, Rothwell JC, Jahanshahi M. Ropinirole, a dopamine agonist with high D3 affinity, reduces proactive inhibition: A double-blind, placebo-controlled study in healthy adults. Neuropharmacol. 2020;179:108278.

118. Scheres A, Oosterlaan J, Swanson J, et al. The effect of methylphenidate on three forms of response inhibition in boys with AD/HD. J Abnorm Child Psychol. 2003;31(1):105-120.

119. Nandam LS, Hester R, Wagner J, et al. Methylphenidate but not atomoxetine or citalopram modulates inhibitory control and response time variability. Biol Psychiatry. 2011;69(9):902-904.

120. Cubillo A, Smith AB, Barrett N, et al. Shared and drug-specific effects of atomoxetine and methylphenidate on inhibitory brain dysfunction in medication-naive ADHD boys. Cereb Cortex. 2014;24(1):174-185.

121. Logemann HNA, Böcker KBE, Deschamps $\mathrm{PKH}$, et al. Haloperidol 2 mg impairs inhibition but not visuospatial attention. Psychopharmacol (Berl.). 2017;234(2):235-244.

122. Eagle DM, Wong JCK, Allan ME, Mar AC, Theobald DE, Robbins TW. Contrasting roles for dopamine D1 and D2 receptor subtypes in the dorsomedial striatum but not the nucleus accumbens core during behavioral inhibition in the stop-signal task in rats. J Neurosci. 2011;31(20):7349-7356.

123. Nandam LS, Hester R, Wagner J, et al. Dopamine D2 receptor modulation of human response inhibition and error awareness. J Cogn Neurosci. 2013;25(4):649-656.

124. Ghahremani DG, Lee B, Robertson CL, et al. Striatal dopamine D2/D3 receptors mediate response inhibition and related activity in frontostriatal neural circuitry in humans. J Neurosci. 2012;32(21):7316-7324.

125. Devoto P, Flore G, Pani L, Gessa GL. Evidence for co-release of noradrenaline and dopamine from noradrenergic neurons in the cerebral cortex. Mol Psychiatry. 2001;6(6):657-664.

126. Devoto P, Flore G, Saba P, Fà M, Gessa GL. Stimulation of the locus coeruleus elicits noradrenaline and dopamine release in the medial prefrontal and parietal cortex. J Neurochem. 2005; 92(2):368-374.

127. Duszkiewicz AJ, McNamara CG, Takeuchi T, Genzel L. Novelty and dopaminergic modulation of memory persistence: A tale of two systems. Trends Neurosci. 2019;42(2):102-114.

128. Varazzani C, San-Galli A, Gilardeau S, Bouret S. Noradrenaline and dopamine neurons in the reward/effort trade-off: A direct electrophysiological comparison in behaving monkeys. $J$ Neurosci. 2015;35(20):7866-7877.

129. Scatton B, Javoy-Agid F, Rouquier L, Dubois B, Agid Y. Reduction of cortical dopamine, noradrenaline, serotonin and their metabolites in Parkinson's disease. Brain Res. 1983;275(2): 321-328.

130. Pifl C, Kish SJ, Hornykiewicz O. Thalamic noradrenaline in Parkinson's disease: Deficits suggest role in motor and nonmotor symptoms. Mov Disord. 2012;27(13):1618-1624.

131. Tsvetanov KA, Ye Z, Hughes L, et al.; Cambridge Centre for Ageing and Neuroscience. Activity and connectivity differences underlying inhibitory control across the adult life span. $J$ Neurosci. 2018;38(36):7887-7900.

132. Koda K, Ago Y, Cong Y, Kita Y, Takuma K, Matsuda T. Effects of acute and chronic administration of atomoxetine and methylphenidate on extracellular levels of noradrenaline, dopamine and serotonin in the prefrontal cortex and striatum of mice. J Neurochem. 2010;114(1):259-270.

133. Ramoz N, Boni C, Downing AM, et al. A Haplotype of the Norepinephrine Transporter (Net) Gene Slc6a2 is associated with clinical response to atomoxetine in attention-deficit hyperactivity disorder (ADHD). Neuropsychopharmacol. 2009; 34(9):2135-2142.

134. Whelan R, Conrod PJ, Poline J-B, et al.; IMAGEN Consortium. Adolescent impulsivity phenotypes characterized by distinct brain networks. Nat Neurosci. 2012;15(6):920-925.

135. Le AN, Ziauddeen H, Fernandez-Egea E, et al. SLC6A2 genotype variation and the modulatory effects of atomoxetine on inhibitory control in individuals with and without cocaine use disorder. Abstr 30th ECNP Congr. 2017;27:S1070-S1071.

136. Sara SJ. The locus coeruleus and noradrenergic modulation of cognition. Nat Rev Neurosci. 2009;10(3):211-223.

137. Oertel WH, Henrich MT, Janzen A, Geibl FF. The locus coeruleus: Another vulnerability target in Parkinson's disease. Mov Disord. 2019;34(10):1423-1429. 